\title{
Rotor Spectra, Berry Phases, and Monopole Fields: from Antiferromagnets to QCD
}

\author{
S. Chandrasekharan ${ }^{a}$, F.-J. Jiang ${ }^{b}$, M. Pepe ${ }^{c}$, and U.-J. Wiese ${ }^{b}$ \\ a Department of Physics, Box 90305, Duke University, Durham, North Carolina 27708 \\ ${ }^{b}$ Institute for Theoretical Physics, Bern University, Sidlerstrasse 5, 3012 Bern, Switzerland \\ ${ }^{c}$ Istituto Nazionale di Fisica Nucleare and Dipartimento di Fisica, \\ Università di Milano-Bicocca, 3 Piazza della Scienza, 20126 Milano, Italy
}

\begin{abstract}
The order parameter of a finite system with a spontaneously broken continuous global symmetry acts as a quantum mechanical rotor. Both antiferromagnets with a spontaneously broken $S U(2)_{s}$ spin symmetry and massless QCD with a broken $S U(2)_{L} \times S U(2)_{R}$ chiral symmetry have rotor spectra when considered in a finite volume. When an electron or hole is doped into an antiferromagnet or when a nucleon is propagating through the QCD vacuum, a Berry phase arises from a monopole field and the angular momentum of the rotor is quantized in half-integer units.

PACS numbers: 75.50.Ee, 12.38.-t, 12.39.Fe, 03.65.Vf
\end{abstract}

Berry phases and monopole fields are familiar from adiabatic processes in quantum mechanical systems [1, 2]. For example, the slow rotation of the nuclei in a diatomic molecule is influenced by a geometric vector potential generated by the fast motion of the electrons [3, 4]. The Abelian and non-Abelian monopole content of these vector potentials was worked out elegantly by Moody, Shapere, and Wilczek [5]. In this paper we discuss Berry phases and monopole fields for rotors arising in condensed matter and particle physics systems with a spontaneously broken continuous global symmetry.

The undoped precursors of layered cuprate hightemperature superconductors are antiferromagnets with a spontaneously broken $S U(2)_{s}$ spin symmetry. When one considers an antiferromagnet of finite volume $V$ at very low temperatures, the dynamics are dominated by the spatially independent zero-mode of the staggered magnetization order parameter

$$
\vec{e}(t)=(\sin \theta(t) \cos \phi(t), \sin \theta(t) \sin \phi(t), \cos \theta(t)),
$$

which represents a slow quantum mechanical rotor governed by the Lagrangian [ 6 ]

$$
\mathcal{L}=\frac{\Theta}{2} \partial_{t} \vec{e} \cdot \partial_{t} \vec{e}=\frac{\Theta}{2}\left[\left(\partial_{t} \theta\right)^{2}+\sin ^{2} \theta\left(\partial_{t} \varphi\right)^{2}\right] .
$$

Integrating out the fast non-zero modes of the staggered magnetization at one loop, and assuming a 2-dimensional quadratic periodic volume, the moment of inertia was determined by Hasenfratz and Niedermayer [ [] as

$$
\Theta=\frac{\rho_{s} V}{c^{2}}\left[1+\frac{3.900265}{4 \pi}\left(\frac{c}{\rho_{s} L}\right)+\mathcal{O}\left(\frac{1}{L^{2}}\right)\right],
$$

where $\rho_{s}$ is the spin stiffness and $c$ is the spinwave velocity. The momenta conjugate to $\theta$ and $\varphi$ are

$$
p_{\theta}=\frac{\delta \mathcal{L}}{\delta \partial_{t} \theta}=\Theta \partial_{t} \theta, p_{\varphi}=\frac{\delta \mathcal{L}}{\delta \partial_{t} \varphi}=\Theta \sin ^{2} \theta \partial_{t} \varphi,
$$

and the resulting Hamiltonian

$$
H=-\frac{1}{2 \Theta}\left(\frac{1}{\sin \theta} \partial_{\theta}\left[\sin \theta \partial_{\theta}\right]+\frac{1}{\sin ^{2} \theta} \partial_{\varphi}^{2}\right)=\frac{\vec{L}^{2}}{2 \Theta}
$$

is just the Laplacian on the sphere $S^{2}$. Correspondingly, the energy spectrum is that of a quantum mechanical rotor with angular momentum $l \in\{0,1,2, \ldots\}$, i.e.

$$
E_{l}=\frac{l(l+1)}{2 \Theta},
$$

with each state being $(2 l+1)$-fold degenerate. The rotor features have been verified in numerical simulations of the antiferromagnetic quantum Heisenberg model [7, 8] (or equivalently of the $t-J$ model at half-filling). It should be noted that a quantum ferromagnet does not behave as a rotor because its order parameter - the uniform magnetization - is a conserved quantity.

When a single hole or electron is doped into the antiferromagnet, the spin of the system changes by $1 / 2$ and thus the angular momentum of the resulting rotor must then be quantized in half-integer units. As we will see, in the language of low-energy effective theories, this half-integer quantization is a result of Berry phases and monopole fields. Systematic low-energy effective theories for charge carriers in an antiferromagnet were recently constructed in [9, 10]. The leading terms in the low-energy Lagrangian of holes or electrons with a small momentum $\vec{p}$ are given by

$$
\mathcal{L}=\frac{\Theta}{2} \partial_{t} \vec{e} \cdot \partial_{t} \vec{e}+\Psi^{\dagger}\left[E(\vec{p})-i \partial_{t}+v_{t}^{3} \sigma_{3}+\lambda V_{t}\right] \Psi .
$$

Here $\Psi(t)=\left(\begin{array}{l}\psi_{+}(t) \\ \psi_{-}(t)\end{array}\right)$ is a two-component Grassmann valued field describing fermions with spin parallel $(+)$ or anti-parallel (-) to the local staggered magnetization. It should be noted that we have suppressed an additional flavor index of the hole fields in a doped cuprate antiferromagnet [10], which distinguishes between holes from different pockets in the Brillouin zone. The fermion energy $E(\vec{p})$ as well as $\lambda$ can be determined by integrating out the non-zero momentum modes of the staggered magnetization, e.g. at one loop. For hole- or electron-doped cuprates as well as for the $t-J$ model it was predicted 
that $\lambda=0$ [10], while for other antiferromagnets in general $\lambda \neq 0[9]$. The Abelian vector potential $v_{t}^{3}(t)$ is the diagonal component of the composite vector field

$$
v_{t}=u \partial_{t} u^{\dagger}=i v_{t}^{a} \sigma_{a}=i v_{t}^{3} \sigma_{3}+i V_{t} .
$$

Here $\sigma_{a}$ are the Pauli matrices and

$$
u=\left(\begin{array}{cc}
\cos \frac{\theta}{2} & \sin \frac{\theta}{2} \exp (-i \varphi) \\
-\sin \frac{\theta}{2} \exp (i \varphi) & \cos \frac{\theta}{2}
\end{array}\right)
$$

represents a transformation which rotates $\vec{e}(t)$ into the 3-direction. One then obtains

$$
\begin{aligned}
v_{t}^{3} & =\sin ^{2} \frac{\theta}{2} \partial_{t} \varphi \\
V_{t} & =\frac{1}{2} \sin \theta\left(\cos \varphi \sigma_{1}+\sin \varphi \sigma_{2}\right) \partial_{t} \varphi \\
& +\frac{1}{2}\left(\sin \varphi \sigma_{1}-\cos \varphi \sigma_{2}\right) \partial_{t} \theta
\end{aligned}
$$

These velocity-dependent terms give rise to a modification of the canonically conjugate momenta such that

$$
\Theta \partial_{t} \theta=p_{\theta}+i A_{\theta}, \Theta \partial_{t} \varphi=\frac{1}{\sin ^{2} \theta}\left(p_{\varphi}+i A_{\varphi}\right)
$$

with the non-Abelian vector potential

$$
\begin{aligned}
& A_{\theta}=i \frac{\lambda}{2}\left(\sin \varphi \sigma_{1}-\cos \varphi \sigma_{2}\right), \\
& A_{\varphi}=i \sin ^{2} \frac{\theta}{2} \sigma_{3}+i \frac{\lambda}{2} \sin \theta\left(\cos \varphi \sigma_{1}+\sin \varphi \sigma_{2}\right),
\end{aligned}
$$

and the corresponding field strength

$$
F_{\theta \varphi}=\partial_{\theta} A_{\varphi}-\partial_{\varphi} A_{\theta}+\left[A_{\theta}, A_{\varphi}\right]=i \frac{1-\lambda^{2}}{2} \sin \theta \sigma_{3}
$$

Remarkably, the resulting geometric Berry gauge field is exactly the same as for a diatomic molecule [5]. For cuprates $(\lambda=0)$ the vector potential is Abelian and describes a monopole with quantized magnetic flux. The path $\vec{e}(t)$ in periodic Euclidean time defines a closed loop $\mathcal{C}$ on $S^{2}$. The Boltzmann factor in the path integral contains a Wilson loop along $\mathcal{C}$ which manifests itself as a Berry phase. Using Stokes' theorem, the Berry phase is given by the magnetic flux enclosed in $\mathcal{C}$. Since the enclosed flux is well-defined only up to the area $4 \pi$ of $S^{2}$, the magnetic charge is $\pm \frac{1}{2}$ as a consequence of the Dirac quantization condition. For a general antiferromagnet $(\lambda \neq 0)$ the vector potential becomes non-Abelian and the flux is no longer quantized.

The resulting Hamilton operator takes the form

$$
\begin{aligned}
H(\lambda) & =-\frac{1}{2 \Theta}\left\{\frac{1}{\sin \theta}\left(\partial_{\theta}-A_{\theta}\right)\left[\sin \theta\left(\partial_{\theta}-A_{\theta}\right)\right]\right. \\
& \left.+\frac{1}{\sin ^{2} \theta}\left(\partial_{\varphi}-A_{\varphi}\right)^{2}\right\}+E(\vec{p})
\end{aligned}
$$

The solution for the energy spectrum can be obtained along the lines of [5]. First, one can show that the Hamiltonian $H(0)$ (with $\lambda=0$ ) commutes with the angular momentum operators

$$
\begin{aligned}
& J_{ \pm}=\exp ( \pm i \varphi)\left( \pm \partial_{\theta}+i \cot \theta \partial_{\varphi}-\frac{1}{2} \tan \frac{\theta}{2} \sigma_{3}\right) \\
& J_{3}=-i \partial_{\varphi}-\frac{\sigma_{3}}{2}
\end{aligned}
$$

and is given by

$$
H(0)=\frac{1}{2 \Theta}\left(\vec{J}^{2}-\frac{1}{4}\right)+E(\vec{p}),
$$

such that the energy spectrum takes the form

$$
E_{j}(0)=\frac{1}{2 \Theta}\left[j(j+1)-\frac{1}{4}\right]+E(\vec{p}) .
$$

Here $j$ is a half-integer. In this case, each state is $2(2 j+1)$-fold degenerate because the fermion sectors + and - cost the same energy. The corresponding wave functions with half-integer angular momentum are monopole harmonics [11, 12]. In particular, the ground state wave functions are

$$
\begin{aligned}
& Y_{\frac{1}{2}, \pm \frac{1}{2}}^{ \pm}(\theta, \varphi)=\frac{1}{\sqrt{2 \pi}} \sin \frac{\theta}{2} \exp ( \pm i \varphi), \\
& Y_{\frac{1}{2}, \mp \frac{1}{2}}^{ \pm}(\theta, \varphi)=\frac{1}{\sqrt{2 \pi}} \cos \frac{\theta}{2} .
\end{aligned}
$$

It should be noted that $Y_{\frac{1}{2}, \frac{1}{2}}^{+}(\theta, \varphi)$ and $Y_{\frac{1}{2},-\frac{1}{2}}^{-}(\theta, \varphi)$ have coordinate singularities at $\theta=\pi$ related to the Dirac string. Following $\mathrm{Wu}$ and Yang [12], one can avoid the coordinate singularity by introducing different coordinate patches glued together by gauge transformations.

The Hamiltonian with $\lambda \neq 0$ takes the form

$$
H(\lambda)=H(0)+\frac{1}{2 \Theta}\left(\lambda C+\frac{1}{2} \lambda^{2}\right),
$$

and still commutes with $\vec{J}$ of eq. $(15)$. Here

$$
\begin{aligned}
C & =-i\left(\sin \varphi \partial_{\theta}+\frac{\cos \varphi}{\sin \theta} \partial_{\varphi}-\frac{1}{2} \sin \varphi \tan \frac{\theta}{2}\right) \sigma_{1} \\
& +i\left(\cos \varphi \partial_{\theta}-\frac{\sin \varphi}{\sin \theta} \partial_{\varphi}-\frac{1}{2} \cos \varphi \tan \frac{\theta}{2}\right) \sigma_{2},
\end{aligned}
$$

and $[C, \vec{J}]=0$. Using $C^{2}=\vec{J}^{2}+\frac{1}{4}$ one obtains the energy spectrum

$$
E_{j}(\lambda)=\frac{1}{2 \Theta}\left[j^{\prime}\left(j^{\prime}+1\right)+\frac{\lambda^{2}-1}{4}\right]+E(\vec{p}),
$$

with $j^{\prime}=j \pm \frac{\lambda}{2}$ and $j$ again being a half-integer. For $\lambda \neq 0$ the fermion sectors + and - get mixed and the previously degenerate $2(2 j+1)$ states are now split into 
two groups of $2 j+1$ degenerate states. Interestingly, for $\lambda= \pm 1$ the monopole field strength of eq.(13) vanishes and $E_{j}( \pm 1)=\frac{1}{2 \Theta} j^{\prime}\left(j^{\prime}+1\right)$ with $j^{\prime}=j \pm \frac{1}{2}$. In that case, the rotor spectrum looks like the one of eq.(6) although the angular momentum $j$ is now a half-integer.

Let us now consider QCD with two massless flavors and thus with a spontaneously broken $S U(2)_{L} \times S U(2)_{R}$ chiral symmetry. When the theory is put in a finite spatial volume $V$, as it is the case in numerical simulations of lattice QCD, the chiral order parameter $U(t) \in S U(2)$ describes a quantum rotor with the Lagrangian

$$
\mathcal{L}=\frac{\Theta}{4} \operatorname{Tr}\left[\partial_{t} U^{\dagger} \partial_{t} U\right]
$$

At tree-level the moment of inertia is given by $\Theta=F_{\pi}^{2} V$ where $F_{\pi}$ is the pion decay constant. The corresponding Hamiltonian is the Laplacian on the sphere $S^{3}$. The QCD rotor spectrum has been derived by Leutwyler [13] in the $\delta$-expansion of chiral perturbation theory [14] as

$$
E_{l}=\frac{j_{L}\left(j_{L}+1\right)+j_{R}\left(j_{R}+1\right)}{\Theta}=\frac{l(l+2)}{2 \Theta} .
$$

In this case, $j_{L}=j_{R}$ with $l=j_{L}+j_{R} \in\{0,1,2, \ldots\}$ and each state is $\left(2 j_{L}+1\right)\left(2 j_{R}+1\right)=(l+1)^{2}$-fold degenerate. The low-energy dynamics of nucleons and pions is described by baryon chiral perturbation theory [15, 16, 17, 18. When a nucleon with small momentum $\vec{p}=|\vec{p}| \vec{e}_{p}$ is propagating in the finite volume the Lagrangian reads

$$
\begin{aligned}
\mathcal{L} & =\frac{\Theta}{4} \operatorname{Tr}\left[\partial_{t} U^{\dagger} \partial_{t} U\right] \\
& +\Psi^{\dagger}\left[E(\vec{p})-i \partial_{t}-i v_{t}-i \lambda\left(\vec{\sigma} \cdot \vec{e}_{p}\right) a_{t}\right] \Psi .
\end{aligned}
$$

Here $\Psi(t)$ is a Pauli spinor with a flavor index distinguishing protons and neutrons and $\frac{\vec{\sigma}}{2}$ is the nucleon spin. At tree level $E(\vec{p})=M+\vec{p}^{2} / 2 M$ and $\lambda=g_{A}|\vec{p}| / M$, where $M$ is the mass and $g_{A}$ is the axial vector coupling of the nucleon. As for the antiferromagnet, the parameters $\Theta, E(\vec{p})$, and $\lambda$ get renormalized by the coupling to non-zero momentum pion modes. In the QCD case $u^{2}=U$ and

$$
v_{t}=\frac{1}{2}\left(u \partial_{t} u^{\dagger}+u^{\dagger} \partial_{t} u\right), a_{t}=\frac{1}{2 i}\left(u \partial_{t} u^{\dagger}-u^{\dagger} \partial_{t} u\right) .
$$

Parameterizing

$$
\begin{aligned}
& U(t)=\cos \alpha(t)+i \sin \alpha(t) \vec{e}_{\alpha}(t) \cdot \vec{\tau} \\
& \vec{e}_{\alpha}(t)=(\sin \theta(t) \cos \varphi(t), \sin \theta(t) \sin \varphi(t), \cos \theta(t)), \\
& \vec{e}_{\theta}(t)=(\cos \theta(t) \cos \varphi(t), \cos \theta(t) \sin \varphi(t),-\sin \theta(t)), \\
& \vec{e}_{\varphi}(t)=(-\sin \varphi(t), \cos \varphi(t), 0),
\end{aligned}
$$

one obtains

$$
\begin{aligned}
& v_{t}=i \sin ^{2} \frac{\alpha}{2}\left(\partial_{t} \theta \vec{e}_{\varphi}-\sin \theta \partial_{t} \varphi \vec{e}_{\theta}\right) \cdot \vec{\tau} \\
& a_{t}=\left(\frac{\partial_{t} \alpha}{2} \vec{e}_{\alpha}+\sin \alpha \frac{\partial_{t} \theta}{2} \vec{e}_{\theta}+\sin \alpha \sin \theta \frac{\partial_{t} \varphi}{2} \vec{e}_{\varphi}\right) \cdot \vec{\tau}
\end{aligned}
$$

Here $\vec{\tau}$ are the Pauli matrices for isospin.

The resulting Hamilton operator takes the form

$$
\begin{aligned}
& H(\lambda)=-\frac{1}{2 \Theta}\left\{\frac{1}{\sin ^{2} \alpha}\left(\partial_{\alpha}-A_{\alpha}\right)\left[\sin ^{2} \alpha\left(\partial_{\alpha}-A_{\alpha}\right)\right]\right. \\
& +\frac{1}{\sin ^{2} \alpha \sin \theta}\left(\partial_{\theta}-A_{\theta}\right)\left[\sin \theta\left(\partial_{\theta}-A_{\theta}\right)\right] \\
& \left.+\frac{1}{\sin ^{2} \alpha \sin ^{2} \theta}\left(\partial_{\varphi}-A_{\varphi}\right)^{2}\right\}+E(\vec{p})
\end{aligned}
$$

with the non-Abelian vector potential

$$
\begin{aligned}
A_{\alpha} & =i \frac{\lambda}{2}\left(\vec{\sigma} \cdot \vec{e}_{p}\right) \vec{e}_{\alpha} \cdot \vec{\tau} \\
A_{\theta} & =i\left(\sin ^{2} \frac{\alpha}{2} \vec{e}_{\varphi}+\frac{\lambda}{2}\left(\vec{\sigma} \cdot \vec{e}_{p}\right) \sin \alpha \vec{e}_{\theta}\right) \cdot \vec{\tau} \\
A_{\varphi} & =i\left(-\sin ^{2} \frac{\alpha}{2} \sin \theta \vec{e}_{\theta}\right. \\
& \left.+\frac{\lambda}{2}\left(\vec{\sigma} \cdot \vec{e}_{p}\right) \sin \alpha \sin \theta \vec{e}_{\varphi}\right) \cdot \vec{\tau}
\end{aligned}
$$

and the corresponding field strength

$$
\begin{aligned}
& F_{\alpha \theta}=i \frac{1-\lambda^{2}}{2} \sin \alpha \vec{e}_{\varphi} \cdot \vec{\tau}, \\
& F_{\theta \varphi}=i \frac{1-\lambda^{2}}{2} \sin ^{2} \alpha \sin \theta \vec{e}_{\alpha} \cdot \vec{\tau}, \\
& F_{\varphi \alpha}=i \frac{1-\lambda^{2}}{2} \sin \alpha \sin \theta \vec{e}_{\theta} \cdot \vec{\tau} .
\end{aligned}
$$

This Berry gauge field is a non-Abelian analog of the Abelian monopole field we encountered for the antiferromagnet. The non-Abelian gauge field again has a coordinate singularity, in this case at $\alpha=\pi$, which corresponds to a Dirac string going through the south pole of $S^{3}$. This indicates that there is a non-Abelian magnetic monopole at the center of $S^{3}$.

The generators of $S U(2)_{L} \otimes S U(2)_{R}$ take the form

$$
\begin{aligned}
\vec{J}_{L} & =\frac{1}{2}(\vec{J}-\vec{K}), \vec{J}_{R}=\frac{1}{2}(\vec{J}+\vec{K}), \\
J_{ \pm} & =\exp ( \pm i \varphi)\left( \pm \partial_{\theta}+i \cot \theta \partial_{\varphi}\right)+\frac{\tau_{ \pm}}{2}, \\
J_{3} & =-i \partial_{\varphi}+\frac{\tau_{3}}{2}, \\
K_{ \pm} & =\exp ( \pm i \varphi)\left(i \sin \theta \partial_{\alpha}+i \cot \alpha \cos \theta \partial_{\theta} \mp \frac{\cot \alpha}{\sin \theta} \partial_{\varphi}\right. \\
& \left.\mp \frac{i}{2} \tan \frac{\alpha}{2} \vec{e}_{\theta} \cdot \vec{\tau}+\frac{1}{2} \tan \frac{\alpha}{2} \cos \theta \vec{e}_{\varphi} \cdot \vec{\tau}\right), \\
K_{3} & =i\left(\cos \theta \partial_{\alpha}-\cot \alpha \sin \theta \partial_{\theta}\right) \\
& -\frac{1}{2} \tan \frac{\alpha}{2} \sin \theta \vec{e}_{\varphi} \cdot \vec{\tau} .
\end{aligned}
$$

The Hamiltonian $H(0)$ (with $\lambda=0$ ) can be written as

$$
H(0)=\frac{1}{2 \Theta}\left(\vec{J}^{2}+\vec{K}^{2}-\frac{3}{4}\right)+E(\vec{p}),
$$


such that the energy spectrum takes the form

$$
E_{j}(0)=\frac{1}{2 \Theta}\left[j(j+2)-\frac{1}{2}\right]+E(\vec{p}) .
$$

In this case, $j_{L}=j_{R} \pm \frac{1}{2}$ and $j=j_{L}+j_{R} \in\left\{\frac{1}{2}, \frac{3}{2}, \ldots\right\}$. Each state is $2\left(j+\frac{1}{2}\right)\left(j+\frac{3}{2}\right)$-fold degenerate because the states with spin up and spin down cost the same energy.

The Hamiltonian with $\lambda \neq 0$ can be written as

$$
H(\lambda)=H(0)+\frac{1}{2 \Theta}\left(\lambda C+\frac{3}{4} \lambda^{2}\right),
$$

and it still commutes with $\vec{J}$ and $\vec{K}$. Here

$$
\begin{aligned}
C & =i\left(\vec{\sigma} \cdot \vec{e}_{p}\right)\left(\vec{e}_{\alpha} \partial_{\alpha}+\frac{1}{\sin \theta} \vec{e}_{\theta} \partial_{\theta}\right. \\
& \left.+\frac{1}{\sin \alpha \sin \theta} \vec{e}_{\varphi} \partial_{\varphi}-\tan \frac{\alpha}{2} \vec{e}_{\alpha}\right) \cdot \vec{\tau}
\end{aligned}
$$

and $[C, \vec{J}]=[C, \vec{K}]=0$. Using $C^{2}=\vec{J}^{2}+\vec{K}^{2}+\frac{3}{4}$ one finally obtains the energy spectrum

$$
E_{j}(\lambda)=\frac{1}{2 \Theta}\left[j^{\prime}\left(j^{\prime}+2\right)+\frac{\lambda^{2}-1}{2}\right]+E(\vec{p}),
$$

with $j^{\prime}=j \pm \frac{\lambda}{2}$, where \pm refers to the spin eigenstates of $\vec{\sigma} \cdot \vec{e}_{p}$ with eigenvalues \pm 1 . Thus we see that for $\lambda \neq 0$ the degeneracy is partly lifted and there are now two groups of $\left(j+\frac{1}{2}\right)\left(j+\frac{3}{2}\right)$-fold degenerate states. Remarkably, for $\lambda= \pm 1$ the non-Abelian field strength of eq.(30) vanishes and $E_{j}( \pm 1)=\frac{1}{2 \Theta} j^{\prime}\left(j^{\prime}+2\right)$ with $j^{\prime}=j \pm \frac{1}{2}$. Just as for an antiferromagnet with $\lambda= \pm 1$, the QCD rotor spectrum then looks like the one of eq.(23) although the system now has fermion number one.

The present study in the $\delta$-regime complements other investigations of finite volume effects in the one-nucleon sector of QCD in the $p$ - 19, 20, 21], $\epsilon$ - 22], and $\epsilon^{\prime}$-regimes [23] of chiral perturbation theory. A comparison of numerical lattice QCD data with finite volume predictions of chiral perturbation theory may lead to an accurate determination of low-energy parameters including $F_{\pi}$ and some of the Gasser-Leutwyler coefficients. Before one can do this in the $\delta$-regime, one must match the volumedependent parameters $\Theta, E(\vec{p})$, and $\lambda$ of the effective quantum mechanics to those of the infinite volume effective theory.

While it is difficult to simulate QCD in the chiral limit, simulations of a single hole in the $t$ - $J$ model with an exact $S U(2)_{s}$ spin symmetry are possible using efficient cluster algorithms. Again, before one can extract the parameters of the systematic effective theory of magnons and holes [10] from a comparison with numerical data, it will be necessary to match the volume-dependent parameters $\lambda$ and $E(\vec{p})$ of the effective quantum mechanics to those of the infinite volume effective theory, e.g. at one loop.
However, even without performing this matching calculation, one can check if indeed $\lambda=0$, as predicted for the $t$ - $J$ model in [10].

The effects discussed here are not limited to antiferromagnets or QCD, but arise for any finite system with a spontaneously broken continuous global symmetry (unless the order parameter is conserved). When a symmetry group $G$ breaks spontaneously to a subgroup $H$, the corresponding order parameter takes values in the coset space $G / H$. In a finite volume the symmetry is restored by a slow rotation of the order parameter. When a single fermion is added to the system, one expects Berry phases resulting from monopole gauge fields residing on the manifold $G / H$, with characteristic effects on the rotor spectrum. It would be interesting to work out these effects for general $G$ and $H$ and for an arbitrary fermion representation. A non-trivial case of physical interest is QCD with three massless flavors for which $G / H=S U(3)$ and the baryons transform as flavor octets.

We have benefitted from discussions with G. Colangelo, S. Dürr, C. Haefeli, P. Hasenfratz, and F. Niedermayer. This work was supported in part by the U.S. Department of Energy grant DE-FG02-05ER41368 and by the Schweizerischer Nationalfonds.

[1] M. Berry, Proc. Roy. Soc. London, Ser. A392 (1984) 45.

[2] B. Simon, Phys. Rev. Lett. 51 (1983) 2167.

[3] G. Herzberg and H. C. Longuet-Higgins, Discuss. Faraday Soc. 35 (1963) 77.

[4] C. Mead, Chem. Phys. 49 (1980) 23; 33.

[5] J. Moody, A. Shapere, and F. Wilczek, Phys. Rev. Lett. 56 (1986) 893.

[6] P. Hasenfratz and F. Niedermayer, Z. Phys. B92 (1993) 91.

[7] U.-J. Wiese and H.-P. Ying, Z. Phys. B93 (1994) 147.

[8] B. B. Beard and U.-J. Wiese, Phys. Rev. Lett. 77 (1996) 5130.

[9] F. Kämpfer, M. Moser, and U.-J. Wiese, Nucl. Phys. B729 (2005) 317.

[10] C. Brügger, F. Kämpfer, M. Moser, M. Pepe, and U.-J. Wiese, cond-mat/0606766, to appear in Phys. Rev. B.

[11] M. Fierz, Helv. Phys. Acta 17 (1944) 27.

[12] T. T. Wu and C. N. Yang, Nucl. Phys. B107 (1976) 365; Phys. Rev. D16 (1977) 1018.

[13] H. Leutwyler, Phys. Lett. B189 (1987) 197.

[14] J. Gasser and H. Leutwyler, Nucl. Phys. B250 (1985) 465.

[15] J. Gasser, M. E. Sainio, and A. Svarc, Nucl. Phys. B307 (1988) 779.

[16] E. Jenkins and A. Manohar, Phys. Lett. B255 (1991) 558.

[17] E. Jenkins, Nucl. Phys. B368 (1992) 190.

[18] V. Bernard, N. Kaiser, J. Kambor, and U.-G. Meissner, Nucl. Phys. B388 (1992) 315.

[19] A. Ali-Khan et al., Nucl. Phys. B689 (2004) 175.

[20] S. R. Beane, Phys. Rev. D70 (2004) 034507.

[21] G. Colangelo, A. Fuhrer, and C. Haefeli, Nucl. Phys. Proc. Suppl. 153 (2006) 41.

[22] P. F. Bedaque, H. W. Griesshammer, and G. Rupak, 
Phys. Rev. D71 (2005) 054015.

[23] W. Detmold and M. Savage, Phys. Lett. B599 (2004) 32. 